\title{
Effects of lifestyle intervention during pregnancy on postpartum diabetes among Chinese women with gestational diabetes
}

\author{
Ninghua $\mathrm{Li}^{1}$ (D) Jinnan $\mathrm{Liu}^{1}$ (D) $\cdot$ Cuiping Zhang ${ }^{2}$ (D) $\cdot$ Gongshu Liu $^{2}$ (D) Junhong Leng $^{2}$ (D) $\cdot$ Leishen Wang ${ }^{2}$ (D) \\ Weiqin $\mathrm{Li}^{2}$ (D) ${\text { Zhijie } \mathrm{Yu}^{3} \text { (D) } \cdot \text { Gang Hu}}^{4}$ (D) - Juliana C. N. Chan ${ }^{5,6}$ (Dilin Yang ${ }^{1,7,8}$
}

Received: 25 July 2020 / Accepted: 16 September 2020 / Published online: 16 October 2020

(C) Springer-Verlag GmbH Germany, part of Springer Nature 2020

Keywords Diabetes - Gestational diabetes - Impaired fasting glucose $\cdot$ Impaired glucose tolerance $\cdot$ Intensive lifestyle intervention $\cdot$ Postpartum

$\begin{array}{ll}\text { Abbreviations } \\ \text { GCT } & \text { Glucose challenge test } \\ \text { GDM } & \text { Gestational diabetes mellitus } \\ \text { IFG } & \text { Impaired fasting glucose } \\ \text { IGT } & \text { Impaired glucose tolerance } \\ \text { LGA } & \text { Large-for-gestational-age } \\ \text { PG } & \text { Plasma glucose } \\ \text { SMBG } & \text { Self-monitoring of blood glucose }\end{array}$

To the Editor: Gestational diabetes mellitus (GDM) affected approximately $12.8 \%$ of pregnancies worldwide in 2019 according to the estimation of the International Diabetes

Ninghua Li and Jinnan Liu contributed equally to this study.

Xilin Yang

yxl@hotmail.com; yangxilin@tmu.edu.cn

1 Department of Epidemiology and Biostatistics, School of Public Health, Tianjin Medical University, Tianjin, China

2 Tianjin Women and Children's Health Centre, Tianjin, China

3 Population Cancer Research Program, Dalhousie University, Halifax, NS, Canada

4 Chronic Disease Epidemiology Laboratory, Pennington Biomedical Research Center, Baton Rouge, LA, USA

5 Department of Medicine and Therapeutics, The Chinese University of Hong Kong, Hong Kong SAR, China

6 Li Ka Shing Institute of Health Science, The Chinese University of Hong Kong, Hong Kong SAR, China

7 Tianjin Center for International Collaborative Research on Environment, Nutrition and Public Health, Tianjin, China

8 Tianjin Key Laboratory of Environment, Nutrition and Public Health, Tianjin, China
Federation [1]. Growing evidence suggests that women with a history of GDM have a markedly increased risk of diabetes later in life [2], but it is still unknown whether intensive management of GDM has benefits for prevention of maternal diabetes at postpartum and later in life. Our group conducted a population-based RCT testing the effectiveness of intensive lifestyle intervention of GDM on the risk of macrosomia and large-for-gestational-age (LGA) in the three-tier antenatal care network of Tianjin, China. We found that such an intervention reduced the risk of macrosomia and LGA in offspring of women with GDM [3]. In the current analysis, we investigate the effect of lifestyle intervention on maternal postpartum diabetes among women with prior GDM.

Details of the study setting, design and participants are reported elsewhere [3]. Briefly, 19,847 pregnant women were enrolled and offered a $50 \mathrm{~g} 1 \mathrm{~h}$ glucose challenge test (GCT) between the 24th and 28th week of gestation, at primary hospitals. Women who had GCT $\geq 7.8 \mathrm{mmol} / 1(n=2921)$ were referred to the Tianjin Women and Children's Health Center (Tianjin, China) to undergo a $75 \mathrm{~g} 2 \mathrm{~h}$ OGTT. Based on the International Association of Diabetes and Pregnancy Study Group's criteria, GDM was diagnosed as: fasting plasma glucose $(\mathrm{PG}) \geq 5.1 \mathrm{mmol} / \mathrm{l} ; 1 \mathrm{~h} \mathrm{PG} \geq 10.0 \mathrm{mmol} / \mathrm{l} ;$ or $2 \mathrm{~h} \mathrm{PG}$ $\geq 8.5 \mathrm{mmol} / \mathrm{l}$. Of the 2921 participants, 1440 pregnant women were diagnosed with GDM. In the previous analysis [3], 734 women were sequentially excluded due to not being invited to participate in the late stage of the fieldwork $(n=52)$, meeting one or more of exclusion criteria $(n=440)$ or receiving unintentional intervention $(n=242)$. Ultimately, 706 women received either intensive care $(n=344)$ or usual care $(n=362)$.

The intensive care intervention included three individualised advice sessions on diet and physical activity and three group education sessions. The details of the intensive care intervention have been published previously [3]. 
Table 1 Clinical and biochemical characteristics of participants

\begin{tabular}{llll}
\hline Variables & $\begin{array}{l}\text { UC } \\
(n=173)\end{array}$ & $\begin{array}{l}\text { IC } \\
(n=218)\end{array}$ & $p$ value \\
\hline
\end{tabular}

At baseline

Age, years

Height, cm

Pre-pregnancy BMI, kg/m²

Systolic blood pressure, $\mathrm{mmHg}$

Diastolic blood pressure, $\mathrm{mmHg}$

Han nationality

Parity $\geq 1$

Family history of diabetes in first degree relatives

Current smoker during pregnancy

Alcohol drinker during pregnancy

OGTT

$$
\begin{aligned}
& \text { Fasting PG, mmol/1 } \\
& 1 \mathrm{~h} \mathrm{PG}, \mathrm{mmol} / 1 \\
& 2 \mathrm{~h} \mathrm{PG}, \mathrm{mmol} / \mathrm{l}
\end{aligned}
$$

Gestational age at OGTT, weeks Insulin use

At follow-up

Postpartum weeks at follow-up

On diet treatment after delivery

Postpartum OGTT

Fasting PG, mmol/1

$2 \mathrm{~h} \mathrm{PG}, \mathrm{mmol} / \mathrm{l}$

Adjusted fasting PG, mean mmol/ $1 \pm \mathrm{SEM}^{\mathrm{c}}$

Adjusted 2 h PG, mean $\mathrm{mmol} / 1 \pm \mathrm{SEM}^{\mathrm{c}}$

Glucose categories

Diabetes

IGT and/or IFG

IGT

IFG

Unadjusted ORs of IC vs UC, OR (95\% CI)

Diabetes

IGT and/or IFG

Adjusted ORs of IC vs UC, OR $(95 \% \text { CI })^{\text {d }}$

\begin{tabular}{|c|c|c|}
\hline $29.4(28.1-31.4)$ & $29.5(27.9-32.0)$ & $0.8288^{\mathrm{a}}$ \\
\hline $162(160-165)$ & $162(158-165)$ & $0.5996^{\mathrm{a}}$ \\
\hline $23.0(20.8-25.3)$ & $22.4(20.8-24.8)$ & $0.2839^{\mathrm{a}}$ \\
\hline $110(100-120)$ & $110(100-120)$ & $0.8374^{\mathrm{a}}$ \\
\hline $70(60-75)$ & $70(60-80)$ & $0.5746^{\mathrm{a}}$ \\
\hline $167(96.5)$ & $211(96.8)$ & $0.8880^{\mathrm{b}}$ \\
\hline $6(3.5)$ & $11(5.0)$ & $0.4474^{\mathrm{b}}$ \\
\hline $41(23.7)$ & $55(25.2)$ & $0.7270^{\mathrm{b}}$ \\
\hline $2(1.2)$ & $4(1.8)$ & $0.6976^{\mathrm{b}}$ \\
\hline $9(5.2)$ & $9(4.1)$ & $0.6148^{\mathrm{b}}$ \\
\hline $5.0 \pm 0.5$ & $5.0 \pm 0.6$ & $0.2725^{\mathrm{a}}$ \\
\hline $10.3(9.4-11.0)$ & $10.3(9.6-11.0)$ & $0.5740^{\mathrm{a}}$ \\
\hline $8.6(7.4-9.4)$ & $8.5(7.7-9.3)$ & $0.7628^{\mathrm{a}}$ \\
\hline $26.1(25.6-27.1)$ & $26.3(25.6-27.4)$ & $0.4788^{\mathrm{a}}$ \\
\hline $6(3.5)$ & $12(5.5)$ & $0.3399^{\mathrm{b}}$ \\
\hline $9.6(7.8-12.0)$ & $9.3(7.6-11.3)$ & $0.2416^{\mathrm{a}}$ \\
\hline $1(0.6)$ & $0(0.0)$ & $0.4340^{\mathrm{b}}$ \\
\hline $5.3(4.9-5.7)$ & $5.2(5.0-5.7)$ & $0.8404^{\mathrm{a}}$ \\
\hline $7.2(6.3-8.5)$ & $7.1(6.1-8.2)$ & $0.2709^{\mathrm{a}}$ \\
\hline $5.29 \pm 1.01$ & $5.28 \pm 1.01$ & 0.8546 \\
\hline \multirow[t]{2}{*}{$7.28 \pm 1.02$} & $7.05 \pm 1.01$ & 0.1341 \\
\hline & & $0.9978^{\mathrm{b}}$ \\
\hline $5(2.9)$ & $7(3.2)$ & \\
\hline $61(35.3)$ & $76(34.9)$ & \\
\hline $56(32.4)$ & $70(32.1)$ & \\
\hline \multirow[t]{5}{*}{$5(2.9)$} & $6(2.8)$ & \\
\hline & $1.11(0.34,3.59)$ & 0.8623 \\
\hline & $0.99(0.65,1.51)$ & 0.9534 \\
\hline & $0.94(0.28,3.11)$ & 0.9154 \\
\hline & $0.91(0.58,1.43)$ & 0.6933 \\
\hline
\end{tabular}

Diabetes

IGT and/or IFG

Data are presented as means $\pm \mathrm{SD}$, median (IQR) or $n(\%)$, unless otherwise stated

${ }^{a}$ Derived from Student's $t$ test or Wilcoxon two-sample test

${ }^{\mathrm{b}}$ Derived from $\chi^{2}$ test or Fisher's exact test

${ }^{\mathrm{c}}$ Analysis of covariance was used to adjust for age, family history of diabetes, and $\log _{10}$-transformed fasting and $2 \mathrm{~h}$ PG during OGTT during pregnancy, and data are expressed as mean \pm SEM

${ }^{\mathrm{d}}$ Generalised logit model was used to obtain ORs of intensive care vs usual care for diabetes and IGT and/or IFG following adjustment for age, family history of diabetes, and fasting and $2 \mathrm{~h}$ PG during OGTT during pregnancy

IC, intensive care; UC, usual care
Briefly, total energy intake (i.e. low energy intake) was recommended to the women in the intensive care group based on pre-pregnancy BMI classification for Chinese adults. All women were asked to engage in at least 30 min of light- to moderate-intensity physical activity daily. The intensive care group was also asked to perform self-monitoring of blood 
glucose (SMBG) with blood glucose control targets of $\geq 3.5-\leq 5.1 \mathrm{mmol} / 1$ for fasting capillary blood glucose, and $\leq 7.0 \mathrm{mmol} / 1$ for $2 \mathrm{~h}$ postprandial capillary blood glucose up to the 36th gestational week or $\leq 8.0 \mathrm{mmol} / \mathrm{l}$ from the 36th gestational week onwards [3]. In contrast, the usual care group was only offered routine prenatal care (one group diabetes education session, without asking them to perform SMBG).

At postpartum, all women enrolled in the RCT were invited to participate in a standard $75 \mathrm{~g} 2 \mathrm{~h}$ OGTT. Diabetes, impaired glucose tolerance (IGT) and impaired fasting glucose (IFG) were defined based on the criteria of World Health Organization (1999) [4]. Ethical approval was obtained from the Ethic Committee of Tianjin Women and Children's Health Care Center and written informed consent was obtained before data collection. The primary trial is registered at ClinicalTrials. gov (ClinicalTrials.gov registration no. NCT01565564).

All analyses were performed using the Statistical Analysis System (release 9.4; SAS Institute, Cary, NC, USA). Continuous variables were compared using Student's $t$ test or Wilcoxon two-sample test and categorical variables were compared using $\chi^{2}$ test or Fisher's exact test where appropriate. Analysis of covariance was used to adjust for age, family history of diabetes, and $\log _{10}$-transformed fasting and $2 \mathrm{~h} \mathrm{PG}$ during OGTT during pregnancy. Generalised logit model was used to obtain unadjusted and adjusted ORs of intensive care vs usual care for diabetes and IGT and/or IFG. The adjustment included age, family history of diabetes, and fasting and $2 \mathrm{~h}$ PG during OGTT during pregnancy.

At a median 9.4 (IQR 7.7-11.7) weeks after delivery, 391 (55.4\%) women completed the postpartum OGTT. The baseline clinical and biochemical characteristics, including maternal age, pre-pregnancy BMI, systolic/diastolic blood pressure, ethnicity, parity, family history of diabetes in first degree relatives, gestational age at OGTT, and fasting and $2 \mathrm{~h}$ PG during OGTT during pregnancy were similar between the intensive care group and the usual care group (Table 1). All these baseline variables were also similar by intervention among women who were lost to follow-up (data not shown). At follow-up, fasting and $2 \mathrm{~h} \mathrm{PG}$ during OGTT were similar in the intensive care and the usual care groups. In the intensive care group $(n=218), 3.2 \%$ of women developed postpartum diabetes and $34.9 \%$ developed postpartum IGT and/or IFG. Similarly, $2.9 \%$ of women in the usual care group $(n=173)$ developed postpartum diabetes and $35.3 \%$ developed postpartum IGT and/or IFG $(p>0.9)$. Because there were significant or borderline differences in some baseline variables between women available and those women lost to follow-up either in the intensive care group and/or in the usual care group (data not shown), we performed multivariable analysis to adjust for these baseline variables. The adjusted postpartum fasting and $2 \mathrm{~h}$ PG values were still similar after the adjustment; the adjusted ORs were $0.94(95 \%$ CI $0.28,3.11)$ for diabetes and $0.91(95 \% \mathrm{CI} 0.58,1.43)$ for IGT and/or IFG.
In this secondary analysis of follow-up data of the RCT, we found that $3.1 \%$ of women with GDM developed diabetes at early postpartum, but lifestyle intervention during pregnancy did not reduce the risk of postpartum diabetes. Consistent with our findings, another RCT $(n=457)$ also reported that treatment with formal nutritional counselling and diet therapy during pregnancy had no significant impact on subsequent diabetes among women with mild GDM [5]. Women with GDM are characterised by a lower pancreatic beta cell function and higher insulin resistance years after delivery [6]. Although intensive lifestyle intervention has been found, to some extent, to increase insulin sensitivity and improve beta cell function [7] and reduce the risk of delivery of a macrosomia and/or LGA infant [3], intensive care may not be enough to safeguard beta cell function from declining during pregnancy or at early postpartum in women with GDM. It is also noted that our antenatal care system did not offer a universal OGTT at early pregnancy to pregnant women and some women with GDM may have had IGT and/or IFG before pregnancy. Although our study found that intervention of GDM did not reduce risk of early postpartum diabetes, its effects on long-term risk of diabetes in women with prior GDM still needs to be tested in future RCTs.

Our study had some limitations. The sample size was not large and the low follow-up rate may introduce potential lostto-follow-up bias because some baseline variables were different between women available and not available for follow-up. However, the adjustment analysis may have partially removed potential confounding effects.

In conclusion, we found that intensive lifestyle intervention during pregnancy did not reduce the risk of postpartum diabetes among women with GDM. Its effects on long-term risk of diabetes needs to be tested in future studies.

\section{Trial registration ClinicalTrials.gov NCT01565564.}

Acknowledgements The authors thank all of the health professionals of Tianjin Antenatal Network (Tianjin, China) for their involvement and contribution to the study.

Data availability The datasets used and/or analysed during the current study are available from the corresponding author on reasonable request.

Funding This project was supported by the National Key Research and Development Program of China (grant no.s: 2018YFC1313900, 2018YFC1313903) and BRIDGES (grant no: LT09-227). BRIDGES is an International Diabetes Federation programme supported by an educational grant from Lilly Diabetes. The study sponsor/funder was not involved in the design of the study; the collection, analysis, and interpretation of data; writing the report; and did not impose any restrictions regarding the publication of the report.

Authors' relationships and activities The authors declare that there are no relationships or activities that might bias, or be perceived to bias, their work. 
Contribution statement XY, ZY, GH and JCNC substantially contributed to the conception and design of the work. CZ, GL, JLeng, LW and WL substantially contributed to acquisition of the data. NL, JLiu and XY analysed the data and drafted the article. CZ, GL, JLeng, LW, WL, ZY, $\mathrm{GH}$ and JCNC revised the article critically for important intellectual content. All authors agreed to submit and publish this manuscript. XY is the guarantor of this manuscript and takes full responsibility for the work as a whole, including the study design, access to the data and decision to submit.

\section{References}

1. Yuen L, Saeedi P, Riaz M et al (2019) Projections of the prevalence of hyperglycaemia in pregnancy in 2019 and beyond: results from the International Diabetes Federation Diabetes Atlas, 9th edition. Diabetes Res Clin Pract 157:107841. https://doi.org/10.1016/j. diabres.2019.107841

2. Daly B, Toulis KA, Thomas N et al (2018) Increased risk of ischemic heart disease, hypertension, and type 2 diabetes in women with previous gestational diabetes mellitus, a target group in general practice for preventive interventions: a population-based cohort study. PLoS Med 15(1):e1002488. https://doi.org/10.1371/journal.pmed. 1002488
3. Yang X, Tian H, Zhang F et al (2014) A randomised translational trial of lifestyle intervention using a 3-tier shared care approach on pregnancy outcomes in Chinese women with gestational diabetes mellitus but without diabetes. J Transl Med 12:290. https://doi.org/ 10.1186/s12967-014-0290-2

4. WHO (1999) Definition, diagnosis and classification of diabetes mellitus and its complications: report of a WHO consultation. Part 1, diagnosis and classification of diabetes mellitus. Available from: https://apps.who.int/iris/handle/10665/66040. Accessed 28 June 2020

5. Casey BM, Rice MM, Landon MB et al (2020) Effect of treatment of mild gestational diabetes on long-term maternal outcomes. Am J Perinatol 37(5):475-482. https://doi.org/10.1055/s-0039-1681058

6. Xiang AH, Kjos SL, Takayanagi M, Trigo E, Buchanan TA (2010) Detailed physiological characterization of the development of type 2 diabetes in Hispanic women with prior gestational diabetes mellitus. Diabetes 59(10):2625-2630. https://doi.org/10.2337/db10-0521

7. Kitabchi AE, Temprosa M, Knowler WC et al (2005) Role of insulin secretion and sensitivity in the evolution of type 2 diabetes in the diabetes prevention program: effects of lifestyle intervention and metformin. Diabetes 54(8):2404-2414. https://doi.org/10.2337/ diabetes.54.8.2404

Publisher's note Springer Nature remains neutral with regard to jurisdictional claims in published maps and institutional affiliations. 